Check for updates

Cite this: Nanoscale Adv., 2019, 1, 132

\title{
Noble metal supported hexagonal boron nitride for the oxygen reduction reaction: a DFT study $\dagger$
}

\author{
Seoin Back ${ }^{a}$ and Samira Siahrostami iD *b
}

\begin{abstract}
Discovering active, stable and cost-effective catalysts for the oxygen reduction reaction (ORR) is of utmost interest for commercialization of fuel cells. Scarce and expensive noble metals such as Pt and Pd are the state-of-the-art active ORR catalysts but suffer from low stability against CO poisoning. Hexagonal boron nitride ( $\mathrm{h}-\mathrm{BN}$ ) is a particularly attractive material due to its low cost and stability; however, it suffers from intrinsic low activity toward the ORR in the pristine form as a result of its inherently low conductivity with a large band gap of $\sim 5.5$ electron volts. During the past few years, several strategies such as using metal supports, metal doping and atomic vacancies have been reported to significantly increase the conductivity, thereby promoting the ORR activity. Herein we use density functional theory calculations to systematically study these strategies for activating inert $\mathrm{h}-\mathrm{BN}$ and further examine the stability against $\mathrm{CO}$ poisoning. We show that noble metals, such as $\mathrm{Ag}, \mathrm{Pd}$, and $\mathrm{Pt}$, require boron (B) or nitrogen $(\mathrm{N})$ vacancies to reasonably activate $\mathrm{h}-\mathrm{BN}$ toward the ORR. For example, Pd supported h-BN with B-vacancies exhibits significantly high ORR activity. All three examined metal supported h-BNs are predicted to be stable against $\mathrm{CO}$ poisoning. These results demonstrate that supporting $\mathrm{h}-\mathrm{BN}$ on noble metals is a promising strategy to increase the stability against $\mathrm{CO}$ poisoning while maintaining high ORR activity.
\end{abstract}

Received 24th June 2018
Accepted 10th October 2018

DOI: $10.1039 / c 8 n a 00059 j$

rsc.li/nanoscale-advances toward the ORR. However, its ORR catalytic activity can be significantly improved by surfactant exfoliation, hetero-atom or metal doping, and deposition on metal supports. ${ }^{18-26}$ Gao et al. investigated the role of different defects for $\mathrm{Cu}$ supported BN and found that a boron vacancy helps in binding ORR intermediates more strongly. ${ }^{27}$

On the other hand, h-BN exhibits other unique properties such as high stability which is favorable for the ORR. In fact, the h-BN sheet could serve as a protective layer for the underneath metal substrate and enhance the stability under ORR conditions. ${ }^{13,28,29}$ Recently, Zhang et al. reported that CO binding energy becomes much weaker on Pt supported boron nitride compared to that on the Pt surface, thereby alleviating the $\mathrm{CO}$ poisoning effect. ${ }^{28} \mathrm{Zuo}$ et al. observed that Pd supported boron nitride retained its ORR activity after 4000 cycles, while the halfwave potential of commercial Pt/C decreased by $33 \mathrm{mV} .^{22}$ These experiments indicate that depositing $\mathrm{h}-\mathrm{BN}$ on metal supports is a promising technique to enhance the stability of noble metal supports while maintaining the activity.

Herein, we systematically analyze the ORR catalytic activity of h-BN sheets supported on different noble metals (h-BN@M (M: $\mathrm{Ag}, \mathrm{Pd}$ and $\mathrm{Pt}$ )) using density functional theory (DFT) calculations. For comparison, we also calculate the ORR activity of h-BN@Cu which has been reported to be as active as a Pt catalyst for the ORR. ${ }^{17}$ We show that all the examined h-BNs supported on noble metals require nitrogen $(\mathrm{N})$ or boron $(\mathrm{B})$ vacancies to show reasonable ORR activity. Particularly,
${ }^{a}$ SUNCAT Center for Interface Science and Catalysis, Department of Chemical Engineering, Stanford University, Stanford, CA 94305, USA

${ }^{b}$ Department of Chemistry, University of Calgary, 2500 University Drive NW, Calgary, Alberta, CanadaT2N 1N4.E-mail: samiras.siahrostami@ucalgary.ca

$\dagger$ Electronic supplementary information (ESI) available. See DOI: 10.1039/c8na00059j 
h-BN@Pd with B vacancies has similar ORR activity to a Pt catalyst, which is in very good agreement with recently reported experimental results. ${ }^{22}$ We also show that the stability of h-BN@M against CO poisoning is much improved compared to that of a Pt catalyst. Finally, we also discuss the calculated ORR activity and stability of transition metals doped in single and double vacancies of the two-dimensional h-BN and show that $\mathrm{Cu}$-doped and Ag-doped at single B-vacancy is significantly active and stable for the ORR.

\section{Computational details}

We performed geometry optimizations and calculated electronic energies using the Vienna Ab Initio Simulation Package $(\mathrm{VASP})^{\mathbf{3 0 , 3 1}}$ and projector augmented wave (PAW) pseudopotentials. ${ }^{32}$ We chose to use the BEEF-vdW ${ }^{33}$ exchange-correlation functional, which was shown to accurately describe both chemisorption and physisorption. ${ }^{34}$ The energy cutoff was set to $500 \mathrm{eV}$, and the convergence criteria for energy (self-consistent iteration) and force (ionic relaxation) were set to $10^{-4} \mathrm{eV}$ and $0.05 \mathrm{eV} \AA^{-1}$, respectively. The bulk geometry of BN was optimized using a $(12 \times 12 \times 4)$ Monkhorst-Pack $k$-point mesh, resulting in a B-N distance of $1.45 \AA$, in agreement with the previous calculations and experiments..$^{35,36} \mathrm{~A}(3 \times 3 \times 1) k$-point mesh was utilized to calculate the energetics of metal-supported boron nitride. Note that we utilized the Quantum Espresso $\operatorname{code}^{37}$ with the BEEF-vdW functional ${ }^{33}$ and ultrasoft pseudopotentials $^{38}$ for h-BN based single atom catalyst calculations. More details can be found in the previous publication. ${ }^{39}$

Among the four metal supports ( $\mathrm{Ag}, \mathrm{Cu}, \mathrm{Pd}$, and $\mathrm{Pt})$, we modeled $(4 \times 4)$ BN supported on three-layered (111) surfaces of Ag, Pd and Pt using a $2(\sqrt{ } 3 \times \sqrt{ } 3)$ supercell, while we modeled $(3 \times 3)$ BN supported on $\mathrm{Cu}(111)$ using a $(3 \times 3)$ supercell to minimize the lattice mismatches (Fig. 1). The resulting lattice mismatches were 2.48, 3.86, 2.07 and $1.79 \%$ for $\mathrm{Ag}, \mathrm{Cu}, \mathrm{Pd}$ and $\mathrm{Pt}$, respectively. We note that the previous theoretical investigation showed that influence of strain effect on the binding energies of ORR intermediates was less than $0.10 \mathrm{eV}$ for $4 \%$ strain of $\mathrm{BN} .{ }^{40}$ During the structural optimizations, atoms in the bottom two layers were kept fixed, while other atoms including adsorbates were fully relaxed. To avoid an artificial interaction between periodic images along the $z$-direction, we included a vacuum spacing of $15 \AA$.

The calculated electronic energies were converted into free energies by adding free energy corrections for adsorbates (zeropoint energy, enthalpy and entropy corrections). The free energy correction was obtained from a harmonic oscillator approximation at $300 \mathrm{~K}$ as implemented in the Atomic Simulation Environment (ASE), and the correction values for $* \mathrm{O}, * \mathrm{OH}$ and *OOH were $0.06 \mathrm{eV}, 0.35 \mathrm{eV}$ and $0.42 \mathrm{eV}$, respectively. We also studied the effect of solvation on the adsorption energies of oxygen intermediates on h-BN@Pd as an example. We included an explicit solvation model containing eight $\mathrm{H}_{2} \mathrm{O}$ molecules in the supercell derived from the minima-hopping method (Fig. S1†). ${ }^{\mathbf{4 1}}$ The calculated solvation stabilization effects for ${ }^{*} \mathrm{OH}$ and $* \mathrm{OOH}$ were found to be negligible $(-0.09$ and $-0.01 \mathrm{eV}$, respectively), compared to the -0.5 and $-0.25 \mathrm{eV}$ on metal surfaces. ${ }^{42}$

\section{Results and discussion}

We begin by considering the adhesion energies of h-BN supported on metals, calculated as $E_{\mathrm{h}-\mathrm{BN} @ \mathrm{M}}-E_{\mathrm{M}}-E_{\mathrm{h}-\mathrm{BN}}$, where $E_{\mathrm{h}-\mathrm{BN} @ \mathrm{M}}, E_{\mathrm{M}}$ and $E_{\mathrm{h}-\mathrm{BN}}$ are electronic energies of the h-BN@M complex, clean metal slabs and h-BN with/without vacancies, respectively. Among the various possibilities, the most stable geometries are chosen for further investigations. For example, a B-vac. prefers to sit on top of the metal atom rather than on

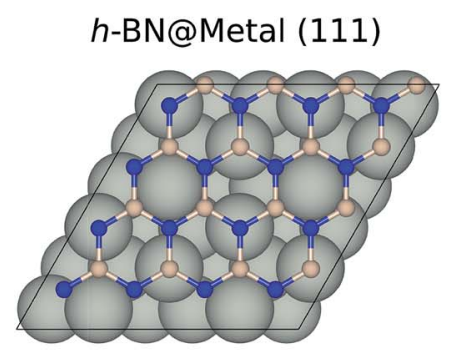

B-vac. $h$-BN@Metal (111)

N-vac. $h$-BN@Metal (111)
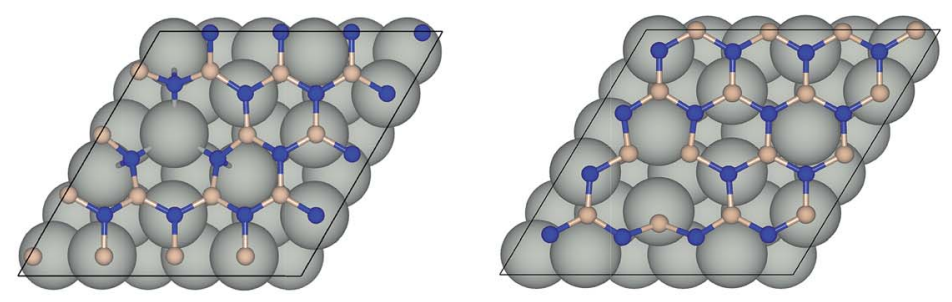

h-BN@Cu (111)

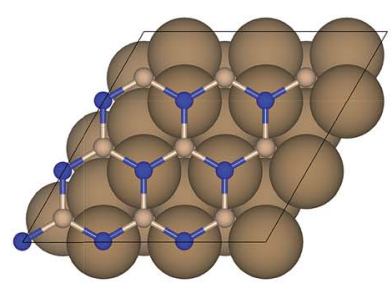

B-vac. $h$-BN@Cu (111)

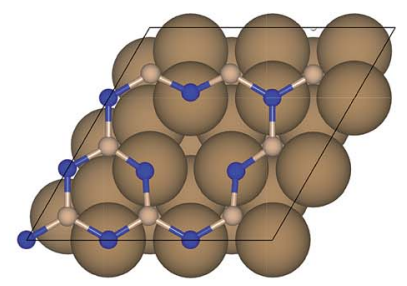

N-vac. h-BN@Cu (111)

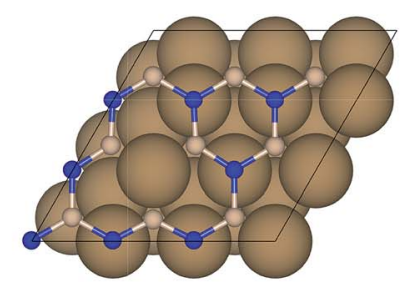

Fig. 1 The optimized geometries of metal supported $B N$ with and without vacancies using a $2(\sqrt{ } 3 \times \sqrt{ } 3$ ) supercell (top) for $\mathrm{Ag}$, $\mathrm{Pd}$, and $\mathrm{Pt}$ and a $(3 \times 3)$ supercell for $\mathrm{Cu}$ (bottom). Blue and pink balls indicate $\mathrm{N}$ and $\mathrm{B}$, respectively. 
Table 1 The calculated adhesion energies of BN supported on metal supports

Adhesion energies per

\begin{tabular}{llll} 
BN pair $(\mathrm{eV})$ & Without vac. & B-vac. & N-vac. \\
\hline $\mathrm{Ag}$ & -0.07 & -0.20 & -0.11 \\
$\mathrm{Pd}$ & -0.08 & -0.34 & -0.29 \\
$\mathrm{Pt}$ & -0.10 & -0.34 & -0.29 \\
$\mathrm{Cu}$ & -0.07 & -0.46 & -0.15
\end{tabular}

the bridge site. Table 1 summarizes the adhesion energies of metal supported h-BN. Although the perfect h-BN binds weakly on metal supports, the interaction becomes stronger when atomic vacancies are introduced. Particularly, adhesion energies of h-BN with B-vacancies are the strongest since $\mathrm{N}$ atoms tend to move toward the metal supports. This is not observed for the case of $\mathrm{N}$-vacancies. We further analyzed the bonding character of h-BN and metal supports using the electron localization function (ELF), ${ }^{43}$ where we found that van der Waals interactions are dominant (Fig. S2 $\dagger$ ).

Next, we calculated the formation energies for B- or $\mathrm{N}$ vacancies $\left(\Delta E_{\mathrm{vac}}\right)$ for all the h-BN@M complexes using eqn (1).

$$
\Delta E_{\mathrm{vac} .}=E_{\mathrm{B} / \mathrm{N}-\mathrm{vac} . \mathrm{h}-\mathrm{BN} @ \mathrm{Metal}}-E_{\mathrm{h}-\mathrm{BN} @ \mathrm{Metal}}+\mu_{\mathrm{B} / \mathrm{N}}
$$

where $E_{\mathrm{B} / \mathrm{N} \text {-vac. h-BN@Metal }}$ and $E_{\mathrm{h}-\mathrm{BN} @ M e t a l}$ are total energies of the h-BN@M system with and without vacancies, respectively, and $\mu_{\mathrm{B} / \mathrm{N}}$ is the chemical potential of a boron or nitrogen atom. At B-rich conditions, we calculated $\mu_{\mathrm{B}}$ as the electronic energy of a boron atom from $\alpha$-rhombohedral boron crystals and $\mu_{\mathrm{N}}$ as $E_{\mathrm{h}-\mathrm{BN}}-\mu_{\mathrm{B}}$, where $E_{\mathrm{BN}}$ is the total energy of formula unit BN. At N-rich conditions, $\mu_{\mathrm{N}}$ is calculated from a $\mathrm{N}_{2}$ molecule, and $\mu_{\mathrm{B}}$ from $E_{\mathrm{h}-\mathrm{BN}}-\mu_{\mathrm{N}}$. For the $\mathrm{Ag}, \mathrm{Pd}$ and Pt supports, the formation of $\mathrm{B}$ or $\mathrm{N}$ vacancies requires a similar amount of energy, while $\mathrm{B}$ vacancy formation is predicted to be much easier on the $\mathrm{Cu}$ support. We particularly note that vacancy formation in h-BN is less energy demanding on metal supports than the freestanding sheet, which could be due to the stabilizing effect of metal supports via charge transfer between metal supports and h-BN. Bader charge and charge density difference analysis on this system revealed that the supported h-BN gains or loses electrons when a B-vac. or $\mathrm{N}$-vac. is formed, respectively while the charge transfer is negligible in the absence of vacancies (Table 2 and Fig. 3). This result implies that the metal supports play major roles in stabilizing defective $\mathrm{h}-\mathrm{BN}$ by providing (gaining) electrons in the case of a B-vac. (N-vac.).

Table 2 Bader charge analysis for metal supported h-BN. Negative values indicate that the $\mathrm{h}-\mathrm{BN}$ sheet gains electrons from the metal supports

\begin{tabular}{llll}
\hline Electrons per BN pair & Without vac. & B-vac. & N-vac. \\
\hline $\mathrm{Ag}$ & 0.00 & -0.09 & 0.03 \\
$\mathrm{Pd}$ & 0.00 & -0.05 & 0.02 \\
$\mathrm{Pt}$ & 0.00 & -0.03 & 0.04 \\
$\mathrm{Cu}$ & 0.00 & -0.14 & 0.03
\end{tabular}

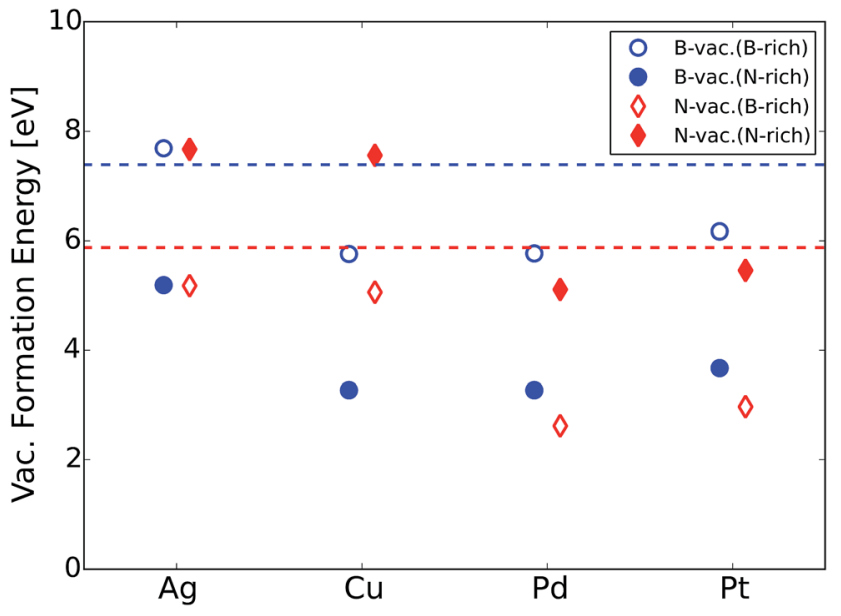

Fig. 2 B or $\mathrm{N}$ atomic vacancy formation energies of metal supported $\mathrm{h}-\mathrm{BN}$ at B-rich and $\mathrm{N}$-rich conditions. The dashed horizontal lines represent $B$ (blue) and $N$ (red) vacancy formation energies of freestanding $\mathrm{h}-\mathrm{BN}$, where only lower values between B-rich and $\mathrm{N}$-rich conditions are shown.

To gain insights into the electronic structure in the presence of vacancies and supports, we calculated the density of states (DOS) projected onto B and $\mathrm{N}$ atoms (Fig. 4 and S3-S5†). In agreement with previous reports in the literature,${ }^{44}$ we observed $\sim 4 \mathrm{eV}$ bandgap for the freestanding h-BN. It is known that metal-induced gap states are formed near the Fermi level for metal supported h-BN, and they significantly influence the catalytic activities. ${ }^{45,46}$ We note that such gap states were observed for all supports particularly with B vacancies (Fig. 4 and S3-S5†).

To investigate the ORR activity, we assume an associative mechanism ${ }^{47}$ and utilize the computational hydrogen electrode (CHE) to include the effect of potential described in detail elsewhere. ${ }^{47,48}$ We calculate the adsorption free energies of ORR intermediates, namely, ${ }^{*} \mathrm{OOH},{ }^{*} \mathrm{O}$, and ${ }^{*} \mathrm{OH}$, relative to gas phase $\mathrm{H}_{2}$ and liquid phase $\mathrm{H}_{2} \mathrm{O}$.

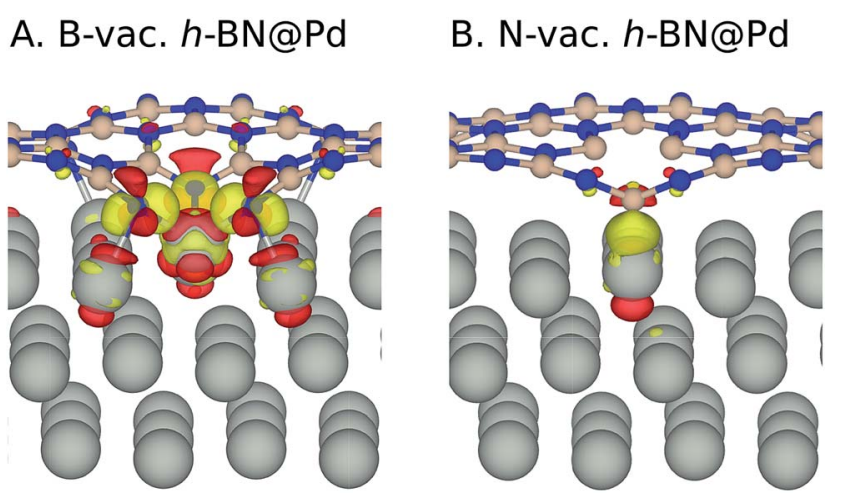

Fig. 3 Charge density difference isosurface upon $\mathrm{h}-\mathrm{BN}$ sheet adsorption on $\operatorname{Pd}\left(\Delta \rho=\rho_{\mathrm{h}-\mathrm{BNaM}}-\rho_{\mathrm{h}-\mathrm{BN}}-\rho_{\mathrm{M}}\right)$. Yellow and red regions indicate the accumulation and depletion of electron charge density, respectively, with the isosurface level set to 0.005 e $\AA^{-3}$. Color code: boron (pink), nitrogen (blue), and metal (silver). 


\section{A. Unsupported BN}

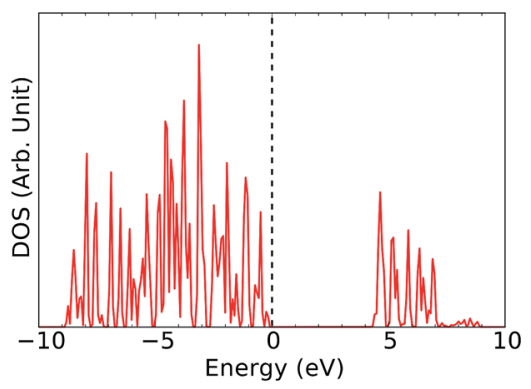

C. B-vac. BN@Pd

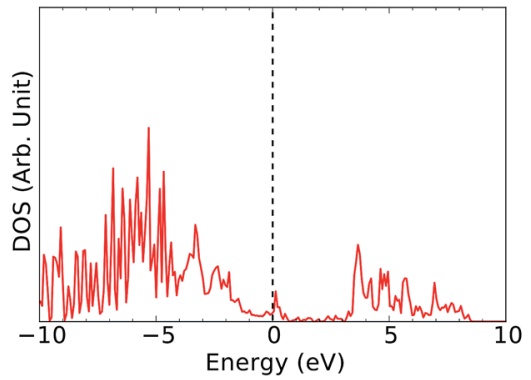

B. BN@Pd

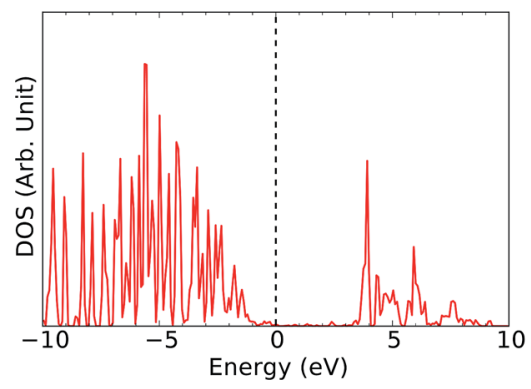

D. N-vac. BN@Pd

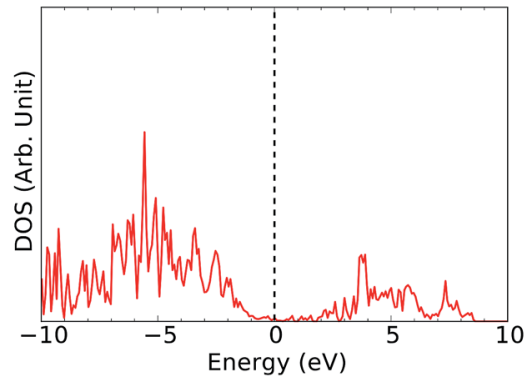

Fig. 4 Projected density of states (PDOS) of the p-orbitals of $B$ and $N$ for (A) unsupported h-BN and (B) Pd supported h-BN without vacancies, (C) with $\mathrm{B}$ vacancies and (D) with $\mathrm{N}$ vacancies. The vertical dashed line represents the position of the Fermi level at $0 \mathrm{eV}$.

$$
\begin{gathered}
\Delta G_{* \mathrm{OOH}}=G(* \mathrm{OOH})+\frac{3}{2} G\left(\mathrm{H}_{2}\right)-G(*)-2 G\left(\mathrm{H}_{2} \mathrm{O}\right) \\
\Delta G^{*} \mathrm{O}=G(* \mathrm{O})+G\left(\mathrm{H}_{2}\right)-G(*)-G\left(\mathrm{H}_{2} \mathrm{O}\right) \\
\Delta G_{* \mathrm{OH}}=G(* \mathrm{OH})+\frac{1}{2} G\left(\mathrm{H}_{2}\right)-G(*)-G\left(\mathrm{H}_{2} \mathrm{O}\right)
\end{gathered}
$$

We note that it is crucial to take a surface coverage under reaction conditions into consideration, which could provide more realistic information rather than assuming a low coverage limit. For example, a catalyst surface with strong *OH adsorption energy is expected to be covered with *OH. For the h-BN@M with/without a B-vac., we found that $\Delta G *^{*} \mathrm{OH}$ is rather weak, so we expected that the low coverage limit provides beneficial insights. However, the binding free energies of the oxygen intermediates on h-BN@M with a N-vac. are significantly strong (up to $-2.0 \mathrm{eV} \Delta G *_{\mathrm{OH}}$ ) and adsorbed *O spontaneously fills the $\mathrm{N}$-vac. during the geometry optimization (Fig. 5B). Therefore, we constructed a surface Pourbaix diagram (more details are in ESI Note $1 \dagger$ ) for all h-BN@M systems with a N-vac. and determined the most stable surface coverage at $0.80 \mathrm{~V}_{\mathrm{RHE}}$, the potential at which ORR current densities are measured (Fig. 5 and S6-S8†). ${ }^{7}$

We now turn our focus on the correlation between binding free energies of ${ }^{*} \mathrm{OH}$ and ${ }^{*} \mathrm{OOH}$. Previous theoretical calculations have revealed a universal scaling relation between $\Delta G{ }^{*} \mathrm{OOH}$ and $\Delta G{ }^{*} \mathrm{OH}$ on various types of catalyst surfaces including transition metals, transition metal oxides and two dimensional materials, resulting in an inherent overpotential $(\sim 0.4 \mathrm{~V})$ for the oxygen reduction and evolution reactions. ${ }^{17,48-50}$ For metal supported h-BN, we also observed a linear scaling behavior between $\Delta G * \mathrm{OH}$ and $\Delta G *^{*} \mathrm{OOH}$, which is similar to the general scaling relation $\left(\Delta G_{*_{\mathrm{OOH}}}=\Delta G_{*_{\mathrm{OH}}}+3.2( \pm 0.2)\right)$. We note that introducing vacancies generally strengthens the binding free energies of reaction intermediates. This in turn improves the ORR activity of h-BN@M systems with weak oxygen binding (such as h-BN@Ag, h-BN@Pd and h-BN@Pt) but adversely affects that of moderate binding h-BN@M catalysts (such as h-BN@Cu) (Fig. 6).

To measure the activity, we take into account the maximum potential at which all the reaction steps are energetically downhill, known as the limiting potential $\left(U_{\mathrm{L}}\right)$. The calculated overpotential is defined as the difference between the limiting potential and the equilibrium potential $(1.23 \mathrm{~V})$. Fig. $6 \mathrm{~B}$ displays the $U_{\mathrm{L}}$ as a function of $\Delta G_{*} \mathrm{OH}$. In agreement with previous theoretical results, h-BN@Cu is expected to catalyze the ORR with an $U_{\mathrm{L}}$ of $0.82 \mathrm{~V} \cdot{ }^{40}$ On the other hand, other metal supports are inactive toward the ORR due to the weak interaction with oxygen intermediates. Once the vacancies are formed, however, all h-BN@M become active for the ORR (Fig. 7). Interestingly, h-BN@Pd with a B-vac. shows a similar activity to Pt (111). Our calculations agree well with a recently published experiment on BN@Pd composites which revealed an ORR activity comparable to that of the commercial $\mathrm{Pt} / \mathrm{C}$ catalyst. ${ }^{22}$

The calculated $\mathrm{CO}$ adsorption energies are $-0.62 \mathrm{eV}$ and -0.01 eV for h-BN@Cu and h-BN@Pd with B-vac., respectively. These energies are significantly weaker than the $\mathrm{CO}$ adsorption energy on Pt (111), -1.65 eV, indicating less CO poisoning on hBN@M compared to Pt (111). 


\section{A. Surface Pourbaix Diagram}

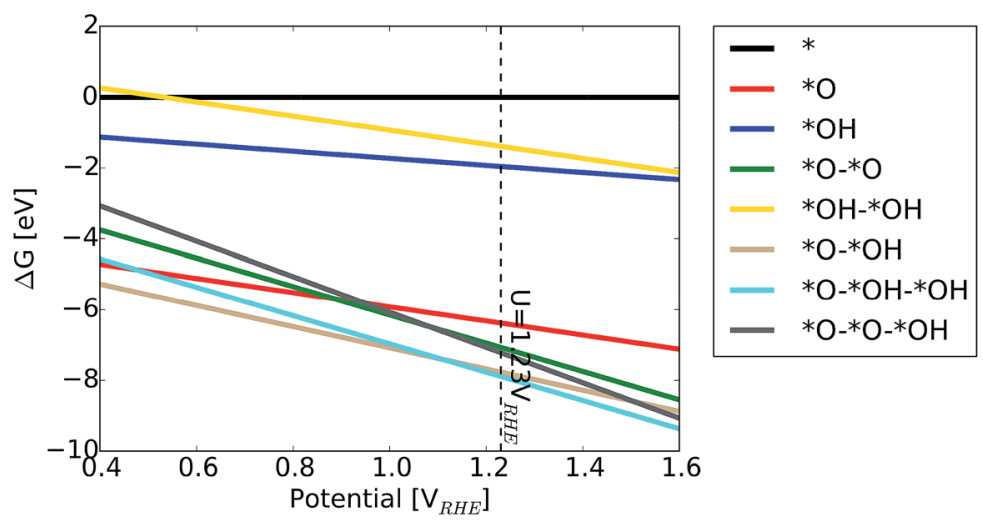

B. ${ }^{*} \mathrm{O}-* \mathrm{OH}$ adsorbed N-vac. $h-\mathrm{BN} @ \mathrm{Pd}$

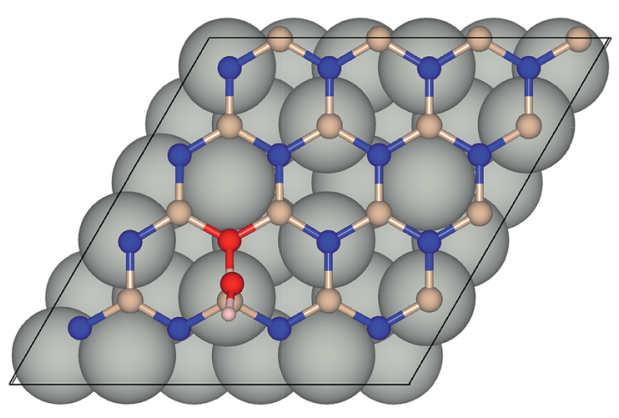

Top View

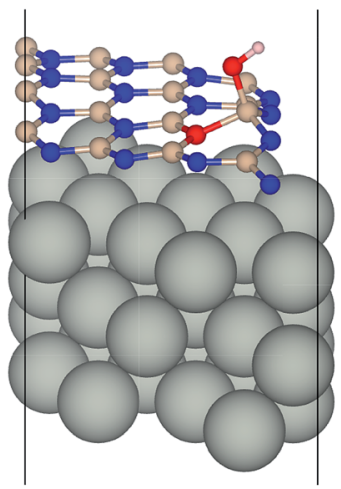

Side View

Fig. 5 (A) Surface Pourbaix diagram of $\mathrm{N}$-vac. BN@Pd. (B) The most stable *O and *OH coadsorbed surface termination of $\mathrm{N}$-vac. BN@Pd at $0.8 \mathrm{~V}_{\mathrm{RHE}}$.

We would like to mention that several experimental efforts have been made to synthesize metal supported BN. For example, the chemical vapor deposition (CVD) technique using borazine $\left(\mathrm{B}_{3} \mathrm{~N}_{3} \mathrm{H}_{6}\right)$ gas has been applied to deposit h-BN sheets on different metal supports. ${ }^{51,52}$ In addition, an exfoliated $\mathrm{BN}$ dispersion can be spin-coated on the metal electrodes. ${ }^{53}$ Vacancies are naturally formed during the synthesis procedure or can be purposely generated using low energy argon or nitrogen ion-bombardment. ${ }^{54}$ Vacancies in h-BN sheets have been successfully observed using an aberration corrected highresolution transmission electron microscope with exit-wave reconstruction. ${ }^{55}$ Given that vacancy formation energies of metal supported BN are notably lower compared to that of the freestanding counterpart (Fig. 2), we expect that atomic

\section{A. Scaling Relation}

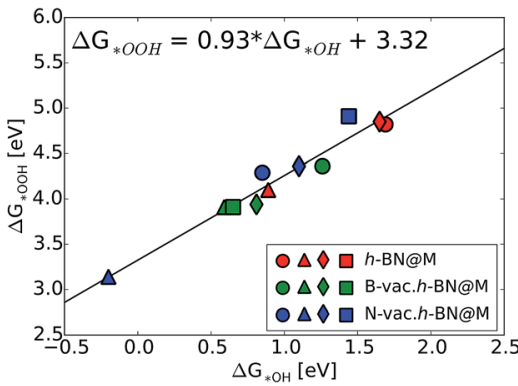

B. Volcano Plot

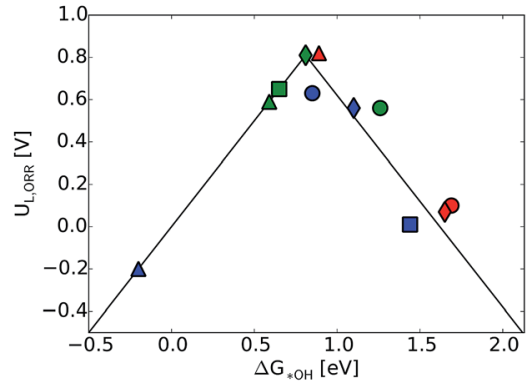

Fig. 6 (A) Scaling relation between $\Delta G_{*_{\mathrm{OH}}}$ and $\Delta G_{* \mathrm{OOH}}$ for different studied h-BN@M complexes. (B) Volcano plot of ORR limiting potential as a function of $\Delta G_{* \mathrm{OH}}$. Circle, triangle, diamond and square symbols indicate $\mathrm{Ag}, \mathrm{Cu}, \mathrm{Pd}$ and Pt supports, respectively. 


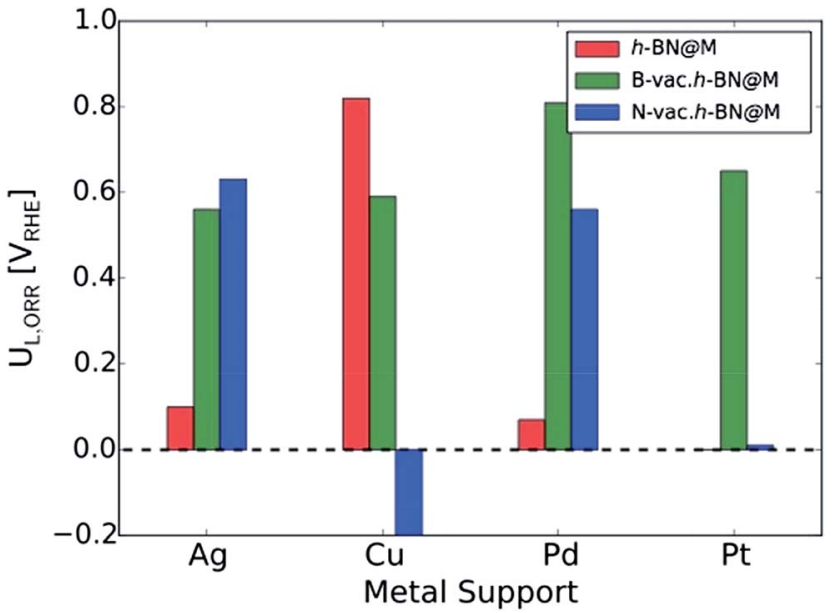

Fig. 7 The calculated ORR limiting potentials $\left(U_{\mathrm{L}, \mathrm{ORR}}\right)$ for metal supported $\mathrm{h}$-BN with/without atomic vacancies. The $\mathrm{h}$-BNaPt is not presented since it does not bind $* \mathrm{OOH}$.

vacancies in metal supported BN are highly probable to form and contribute to the ORR.

Lastly, we would like to emphasize that an alternative strategy to activate pristine two-dimensional materials such as $\mathrm{h}$-BN is to embed single atoms of transition metals in the vacancies. We have recently examined this approach for a wide range of metal atoms implanted in various two-dimensional materials (SACs, single atom catalysts). ${ }^{39}$ Transition metals can be embedded in the single and double vacancies of h-BN (Fig. 8A). By calculating the formation energies $\left(\Delta E_{\mathrm{form}}\right)$ of the metal-doped h-BN systems relative to the bulk metal and h-BN with vacancies, we found strong stabilization between a wide range of transition metal atoms and the h-BN supports with metals doped in the single boron vacancy (B-vac.) being the most stable structures. We also examined the stability of these systems against dissolution under electrochemical ORR conditions. Fig. 8B displays the calculated dissolution potentials of metals as a function of formation energies of metal doped h-BN systems. The horizontal dashed line at $U=0.8 \mathrm{~V}_{\mathrm{RHE}}$ takes into account the potential at which the current density is measured for the ORR. The vertical dashed line represents $\Delta E_{\text {form }}=0.0 \mathrm{eV}$, which is the upper bound to the stability of the systems. The left side of the vertical dashed line indicates a favorable formation of the metal-doped h-BN system, while the upper side of the horizontal dashed line indicates electrochemical stability of the system under ORR conditions. Therefore, catalysts in the pink region are expected to survive under the ORR potential (Fig. 8B). Based on the calculated limiting potentials, doping $\mathrm{Ag}$ and $\mathrm{Cu}$ in the B-vac. of the h-BN sheet provides promising platforms for efficient ORR catalysis with $U_{\mathrm{L}}$ of $0.74 \mathrm{~V}$ and $0.89 \mathrm{~V}$, respectively. This compares very well with those of the two most active ORR systems in the current study, i.e., h-BN@Cu and h-BN@Pd with a B-vac.

\section{Conclusion}

In summary, we have systematically investigated the ORR activity of Ag, Pt and Pd supported h-BNs with/without B and N vacancies. We found that noble metal supports stabilize the h-BN sheet through charge transfer and activate it for the ORR,

\section{A. Single Atom Catalysts}

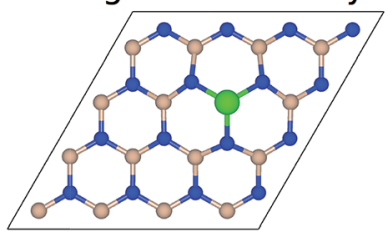

B-SV

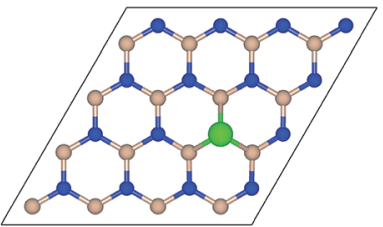

N-SV

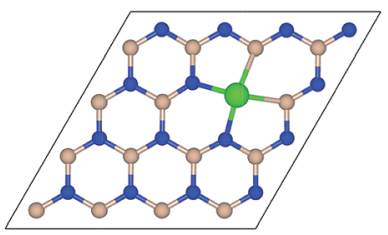

DV
B. Stability Analysis

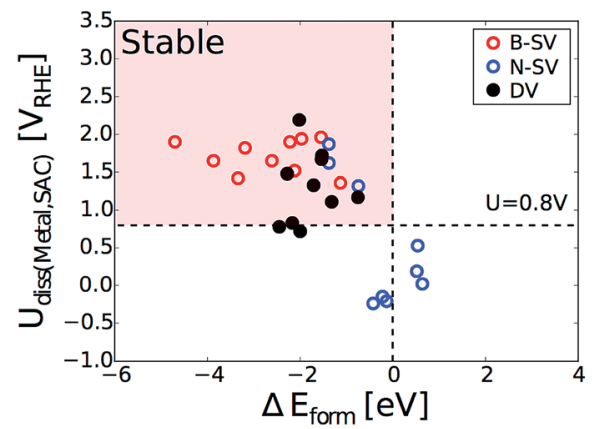

C. ORR Activity

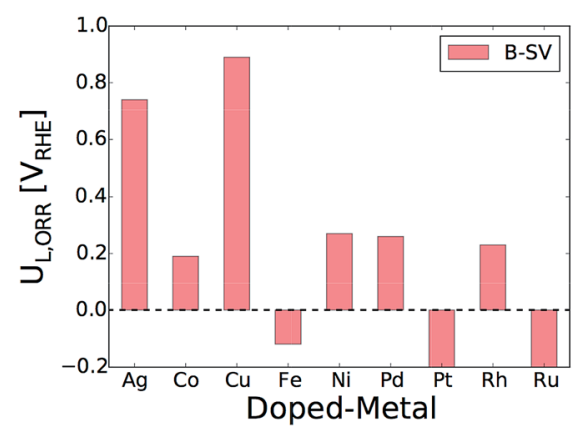

Fig. 8 (A) Top view of $\mathrm{h}-\mathrm{BN}$ based single atom catalysts. B-SV, N-SV, and DV indicate a boron and a nitrogen single vacancy and a double vacancy, respectively. (B) Dissolution potentials vs. formation energies of metal atoms doped in the vacancies of $h$-BN. Catalysts in the pink region are stable in terms of both formation energy and dissolution potential. The details of the calculations can be found in the previous publication. ${ }^{39}$ (C) The calculated ORR limiting potentials $\left(U_{\mathrm{L}}\right)$ for several examined transition metals doped in the B-vac. of h-BN. Note that we only considered B-SV, since it is the most stable configuration. Color code: boron (pink), nitrogen (blue), and metal (green). 
which is inert otherwise. Due to the metal supports and vacancies in the h-BN sheet, additional electronic states appear between occupied and unoccupied states, which are known to be responsible for activating the h-BN sheet. We found that h-BN@Ag, h-BN@Pd, and h-BN@Pt become active only if a B- or $\mathrm{N}$-vac. is introduced. We demonstrated that the h-BN@Cu does not require vacancies and even without vacancies it is a highly active ORR catalytic system with $U_{\mathrm{L}}=0.82 \mathrm{~V}$. This is in agreement with a previous report on the ORR activity of h-BN@Cu. ${ }^{17}$ Our calculated limiting potential for h-BN@Pd with a B-vac. agrees well with the recently reported experimental ORR onset potential of h-BN supported on $\mathrm{Pd} .{ }^{22}$ We also found very weak CO binding energy on h-BN@Pd, in agreement with reported enhanced durability for the ORR. ${ }^{22}$ Lastly, by examining the stability and activity of a wide range of transition metals doped in single and double vacancies of h-BN, we showed that both $\mathrm{Ag}$ - and $\mathrm{Cu}$-doped h-BN can be highly active and stable toward the ORR.

\section{Conflicts of interest}

There are no conflicts to declare.

\section{References}

1 J. Baschuk and X. Li, Int. J. Energy Res., 2001, 25, 695-713.

2 E. Antolini, J. R. Salgado and E. R. Gonzalez, J. Power Sources, 2006, 160, 957-968.

3 M. K. Debe, Nature, 2012, 486, 43.

4 H. A. Gasteiger, S. S. Kocha, B. Sompalli and F. T. Wagner, Appl. Catal., B, 2005, 56, 9-35.

5 V. R. Stamenkovic, B. S. Mun, K. J. Mayrhofer, P. N. Ross and

N. M. Markovic, J. Am. Chem. Soc., 2006, 128, 8813-8819.

6 V. R. Stamenkovic, B. Fowler, B. S. Mun, G. Wang, P. N. Ross,

C. A. Lucas and N. M. Marković, Science, 2007, 315, 493-497.

7 J. Greeley, I. Stephens, A. Bondarenko, T. P. Johansson, H. A. Hansen, T. Jaramillo, J. Rossmeisl, I. Chorkendorff and J. K. Nørskov, Nat. Chem., 2009, 1, 552.

8 S. Back and Y. Jung, ChemCatChem, 2017, 9, 3173-3179.

9 A. L. Strickler, A. Jackson and T. F. Jaramillo, ACS Energy Lett., 2016, 2, 244-249.

10 D. Wang, Y. Yu, H. L. Xin, R. Hovden, P. Ercius, J. A. Mundy, H. Chen, J. H. Richard, D. A. Muller and F. J. DiSalvo, Nano Lett. , 2012, 12, 5230-5238.

11 K. Jiang, Q. Shao, D. Zhao, L. Bu, J. Guo and X. Huang, Adv. Funct. Mater., 2017, 26, 1700830.

12 R. Chattot, T. Asset, P. Bordet, J. Drnec, L. Dubau and F. Maillard, ACS Catal., 2016, 7, 398-408.

13 K. Gong, F. Du, Z. Xia, M. Durstock and L. Dai, Science, 2009, 323, 760-764.

14 Y. Zheng, Y. Jiao, L. Ge, M. Jaroniec and S. Z. Qiao, Angew. Chem., 2013, 125, 3192-3198.

15 L. Qu, Y. Liu, J.-B. Baek and L. Dai, ACS Nano, 2010, 4, 13211326.

16 J. W. To, J. W. D. Ng, S. Siahrostami, A. L. Koh, Y. Lee, Z. Chen, K. D. Fong, S. Chen, J. He and W.-G. Bae, Nano Res., 2017, 10, 1163-1177.
17 S. Siahrostami, C. Tsai, M. Karamad, R. Koitz, M. GarcíaMelchor, M. Bajdich, A. Vojvodic, F. Abild-Pedersen, J. K. Nørskov and F. Studt, Catal. Lett., 2016, 146, 1917-1921. 18 Y. Li, M. Li, L. Jiang, L. Lin, L. Cui and X. He, Phys. Chem. Chem. Phys., 2014, 16, 23196-23205.

19 W. Yang, T.-P. Fellinger and M. Antonietti, J. Am. Chem. Soc., 2010, 133, 206-209.

20 D. S. Su, S. Perathoner and G. Centi, Chem. Rev., 2013, 113, 5782-5816.

21 Z. W. Liu, F. Peng, H. J. Wang, H. Yu, W. X. Zheng and J. Yang, Angew. Chem., 2011, 123, 3315-3319.

22 L.-X. Zuo and L.-P. Jiang, J. Electrochem. Soc., 2017, 164, H805-H811.

23 R. He, D. Wen, W. Shen and M. Li, Phys. Chem. Chem. Phys., 2018, 20, 10240-10246.

24 A. F. Khan, M. P. Down, G. C. Smith, C. W. Foster and C. E. Banks, J. Mater. Chem. A, 2017, 5, 4103-4113.

25 D. Shin, S. Sinthika, M. Choi, R. Thapa and N. Park, ACS Catal., 2014, 4, 4074-4080.

26 G. Elumalai, H. Noguchi and K. Uosaki, Phys. Chem. Chem. Phys., 2014, 16, 13755-13761.

27 X. Gao, S. Wang and S. Lin, ACS Appl. Mater. Interfaces, 2016, 8, 24238-24247.

28 Y. Zhang, X. Weng, H. Li, H. Li, M. Wei, J. Xiao, Z. Liu, M. Chen, Q. Fu and X. Bao, Nano Lett., 2015, 15, 36163623.

29 Y. Tang, B. L. Allen, D. R. Kauffman and A. Star, J. Am. Chem. Soc., 2009, 131, 13200-13201.

30 G. Kresse and D. Joubert, Phys. Rev. B: Condens. Matter Mater. Phys., 1999, 59, 1758.

31 G. Kresse and J. Furthmüller, Comput. Mater. Sci., 1996, 6, 15-50.

32 P. E. Blöchl, Phys. Rev. B: Condens. Matter Mater. Phys., 1994, 50, 17953.

33 J. Wellendorff, K. T. Lundgaard, A. Møgelhøj, V. Petzold, D. D. Landis, J. K. Nørskov, T. Bligaard and K. W. Jacobsen, Phys. Rev. B: Condens. Matter Mater. Phys, 2012, 85, 235149.

34 J. Wellendorff, T. L. Silbaugh, D. Garcia-Pintos, J. K. Nørskov, T. Bligaard, F. Studt and C. T. Campbell, Surf. Sci., 2015, 640, 36-44.

35 A. Du, Y. Chen, Z. Zhu, R. Amal, G. Q. Lu and S. C. Smith, J. Am. Chem. Soc., 2009, 131, 17354-17359.

36 W. Paszkowicz, J. Pelka, M. Knapp, T. Szyszko and S. Podsiadlo, Appl. Phys. A, 2002, 75, 431-435.

37 P. Giannozzi, S. Baroni, N. Bonini, M. Calandra, R. Car, C. Cavazzoni, D. Ceresoli, G. L. Chiarotti, M. Cococcioni and I. Dabo, J. Phys.: Condens. Matter, 2009, 21, 395502.

38 D. Vanderbilt, Phys. Rev. B: Condens. Matter Mater. Phys., 1990, 41, 7892.

39 S. Back, A. Kulkarni and S. Siahrostami, ChemCatChem, 2018, 10, 1-7.

40 R. Koitz, J. K. Nørskov and F. Studt, Phys. Chem. Chem. Phys., 2015, 17, 12722-12727.

41 A. A. Peterson, Top. Catal., 2014, 57, 40-53.

42 Z.-D. He, S. Hanselman, Y.-X. Chen, M. T. Koper and F. Calle-Vallejo, J. Phys. Chem. Lett., 2017, 8, 2243-2246. 
43 A. D. Becke and K. E. Edgecombe, J. Chem. Phys., 1990, 92, 5397-5403.

44 V. Solozhenko, A. Lazarenko, J.-P. Petitet and A. Kanaev, J. Phys. Chem. Solids, 2001, 62, 1331-1334.

45 S. Lin, J. Huang and X. Gao, Phys. Chem. Chem. Phys., 2015, 17, 22097-22105.

46 A. Lyalin, A. Nakayama, K. Uosaki and T. Taketsugu, J. Phys. Chem. C, 2013, 117, 21359-21370.

47 J. K. Nørskov, J. Rossmeisl, A. Logadottir, L. Lindqvist, J. R. Kitchin, T. Bligaard and H. Jonsson, J. Phys. Chem. B, 2004, 108, 17886-17892.

48 A. Kulkarni, S. Siahrostami, A. Patel and J. Nørskov, Chem. Rev., 2018, 118, 2302-2312.

49 I. C. Man, H. Y. Su, F. Calle-Vallejo, H. A. Hansen, J. I. Martínez, N. G. Inoglu, J. Kitchin, T. F. Jaramillo,
J. K. Nørskov and J. Rossmeisl, ChemCatChem, 2011, 3, 1159-1165.

50 V. Viswanathan, H. A. Hansen, J. Rossmeisl and J. K. Nørskov, ACS Catal., 2012, 2, 1654-1660.

51 C. Oshima and A. Nagashima, J. Phys.: Condens. Matter, 1997, 9, 1.

52 A. Preobrajenski, A. Vinogradov and N. Mårtensson, Surf. Sci., 2005, 582, 21-30.

53 K. Uosaki, G. Elumalai, H. Noguchi, T. Masuda, A. Lyalin, A. Nakayama and T. Taketsugu, J. Am. Chem. Soc., 2014, 136, 6542-6545.

54 R. Peter, A. Bozanic, M. Petravic, Y. Chen, L.-J. Fan and Y.-W. Yang, J. Appl. Phys., 2009, 106, 083523.

55 C. Jin, F. Lin, K. Suenaga and S. Iijima, Phys. Rev. Lett., 2009, 102, 195505. 